\title{
Pharmacokinetics, Pharmacodynamics and Safety of Belimumab in Chinese Patients with Systemic Lupus Erythematosus: A Phase I, Open-Label Study
}

\author{
Jing Zhang · Weiguo Wan · Liyan Miao · Jian Wu · Jun Dong • \\ Yinghua Shen · Cui Xiong - Chao Li · Yu Xue - Guoying Cao • \\ Peiming $\mathrm{Ma}$ (D)
}

Received: November 25, 2019 / Published online: January 17, 2020

(c) The Author(s) 2020

\begin{abstract}
Introduction: The B cell survival factor B lymphocyte stimulator (BLyS) is elevated in patients with systemic lupus erythematosus (SLE) and associated with disease activity. Belimumab, a monoclonal antibody specific for soluble BLyS, is approved for the treatment of adults with active, autoantibody-positive SLE receiving standard
\end{abstract}

Enhanced Digital Features To view enhanced digital features for this article go to https://doi.org/10.6084/ m9.figshare.11498760.

Electronic Supplementary Material The online version of this article (https://doi.org/10.1007/s40744020-00193-9) contains supplementary material, which is available to authorized users.

J. Zhang · G. Cao

Phase I Clinical Trial Center, Huashan Hospital Affiliated to Fudan University, Shanghai, China

W. Wan · Y. Xue

Department of Rheumatology, Huashan Hospital Affiliated to Fudan University, Shanghai, China

L. Miao $\cdot$ J. Wu

The National Institution of Drug Clinical Trial \& Laboratory of Phase I Clinical Study, The First Affiliated Hospital of Soochow University, Suzhou, Jiangsu, China

J. Dong $\cdot$ Y. Shen $\cdot$ C. Xiong $\cdot$ P. Ma $(\bowtie)$

GlaxoSmithKline, Shanghai, China

e-mail: peiming.p.ma@gsk.com

C. Li

Novartis Pharma, Shanghai, China therapy. Ethnicity is one of the factors that can potentially affect the pharmacokinetics (PK) of therapeutic monoclonal antibodies, and therefore their efficacy and safety.

Methods: This phase 1, open-label study (200909) evaluated the pharmacokinetics (PK, primary objective), pharmacodynamics (PD), and safety (secondary objectives) of belimumab in Chinese patients with SLE $(N=20)$. Blood samples were taken up to 84 days after a single intravenous (IV) dose of belimumab $10 \mathrm{mg} / \mathrm{kg}$.

Results: Peak serum concentrations of belimumab $\left(C_{\max }\right)$ were obtained within the 1-h infusion. Geometric mean $C_{\max }$, area under the concentration-time curve (time 0 to last quantifiable concentration), terminal half-life, systemic clearance, and volume of distribution were $221 \mu \mathrm{g} / \mathrm{mL}, \quad 2395$ day. $\mu \mathrm{g} / \mathrm{mL}, \quad 14.6$ days, $4.06 \mathrm{~mL} /$ day $/ \mathrm{kg}$, and $85.7 \mathrm{~mL} / \mathrm{kg}$, respectively. Decreases in $\mathrm{CD} 20^{+}, \mathrm{CD} 20^{+} / \mathrm{CD} 27^{-}$naïve, $\mathrm{CD} 20^{+} / \mathrm{CD} 69^{+}$active, $\mathrm{CD} 20^{+} / \mathrm{CD} 138^{+}$plasmacytoid, $\mathrm{CD} 19^{+} / \mathrm{CD} 27^{\mathrm{BRIGHT}} / \mathrm{CD} 38^{\mathrm{BRIGHT}}$ SLE subset, and $\mathrm{CD}^{2} 0^{-} / \mathrm{CD} 138^{+}$plasma $\mathrm{B}$ Cells post-dose were accompanied by an increase in $\mathrm{CD} 20^{+}$/ $\mathrm{CD} 27^{+}$memory B cells. Four cases of upper respiratory tract infection (three mild, one moderate) and one case of mild pharyngitis were possibly drug-related; all resolved during the study.

Conclusion: PK of a single belimumab $10 \mathrm{mg} / \mathrm{kg}$ IV dose in Chinese patients with SLE were similar to those observed previously in Japanese and American (white and African-American) patients with SLE. PD results were consistent with 
belimumab inhibiting BLyS, and belimumab was well tolerated. These data support the use of belimumab in Chinese patients with SLE.

Trial Registration: ClinicalTrials.gov identifier, NCT02880852.

Keywords: Belimumab; Chinese ethnicity; Phar macodynamics; Pharmacokinetics; Systemic lupus erythematosus

\section{Key Summary Points}

\section{Why carry out this study?}

Belimumab, a monoclonal antibody specific for soluble B cell survival factor B lymphocyte stimulator, is approved for the treatment of adults with active, autoantibody-positive systemic lupus erythematosus (SLE) who are receiving standard therapy

Ethnic factors have the potential to affect the pharmacokinetics, and therefore the efficacy and safety, of therapeutic monoclonal antibodies, such as belimumab

In this phase 1 , open-label study, we evaluated the pharmacokinetics, pharmacodynamics, and safety of belimumab in Chinese patients with SLE, to determine if there were any differences to the known profiles from the phase 3 studies of belimumab in Japanese and American (white or African-American) patients with SLE

\section{What was learned from the study?}

In this study, the pharmacokinetics of a single intravenous dose of belimumab $10 \mathrm{mg} / \mathrm{kg}$ in Chinese patients with SLE were similar to those seen previously in the phase 3 studies

Observed changes in the concentrations of all B cell subsets tested confirm the known ability of belimumab to bind and inhibit BLyS, and belimumab was well tolerated

These data support the use of belimumab in Chinese patients with SLE

\section{INTRODUCTION}

Systemic lupus erythematosus (SLE) is a chronic autoimmune disorder that involves multiple organs and presents a myriad of disease manifestations [1]. In the Asia-Pacific region, incidences of SLE per 100,000 person-years range from 2.8 in South Korea to 3.1-6.7 in Chinese populations and 3.3-8.4 in Taiwan [2-4]. These incidences are similar to those in populations from European countries (Denmark 2.35, France 3.32, Iceland 3.3, UK 3.0-4.9) and the Caribbean (Barbados 6.3, Curaçao 4.6, Martinique 4.7), but are less than those in the USA (7.223.2) [4]. Asian patients with SLE have been reported to have more severe disease, significantly higher mean and maximum disease activity, persistently active disease, a higher incidence of renal involvement, and a higher proportion of autoantibody positivity than non-Asian patients with SLE [5]. The 5-year survival rate for SLE in China is 94\%, and the 10 -year survival rate is $89 \%$, similar to other populations in the Asia-Pacific region [5].

Belimumab is a monoclonal antibody that specifically targets the soluble form of B lymphocyte stimulator (BLyS), also known as B cell activating factor, a B cell survival factor. BLyS levels are elevated in patients with SLE, and there is an association between plasma BLyS levels and SLE disease activity [6]. Intravenous (IV) administration of belimumab $10 \mathrm{mg} / \mathrm{kg}$ is approved in more than 70 countries and regions, including Canada, Europe, Japan, and the USA, for the treatment of adults with active, autoantibodypositive SLE receiving standard SLE therapy [6]. Belimumab is also the first treatment approved in children with SLE [7]. The approval of belimumab for adults in Japan was based on the results of two pivotal phase 3 studies, including a study undertaken in Northeast Asia $[6,8]$. This phase 3 , randomized, double-blind study (BEL113750; NCT01345253) was conducted in China, Japan, and South Korea to assess the efficacy and safety of belimumab $10 \mathrm{mg} / \mathrm{kg}$ IV every 4 weeks for 48 weeks, compared with placebo, both as add-on to standard of care in patients with SLE, $77 \%$ of whom were from China [8]. In this study, belimumab significantly 
reduced disease activity with a safety profile similar to that of placebo [8].

The pharmacokinetics (PK) and pharmacodynamics (PD) of IV administration of belimumab have previously been investigated in patients with SLE from Japan [9] and the USA (white or African-American) [10], and were generally similar across these populations. After administration of a single dose of belimumab $10 \mathrm{mg} / \mathrm{kg}$ IV in Japanese or American patients with SLE, the terminal half-life $\left(t_{1 / 2}\right)$ was 15.7 and 10.6 days, the volume of distribution $\left(V_{\mathrm{z}}\right)$ was 76.2 and $86.3 \mathrm{~mL} / \mathrm{kg}$, and mean clearance (CL) was 3.6 and $6.9 \mathrm{~mL} /$ day $/ \mathrm{kg}$, respectively $[9,10]$.

Ethnicity is one of several interwoven factors known to underlie variability in PK profiles between individuals receiving therapeutic monoclonal antibodies. Alterations in monoclonal antibody clearance have been observed between differing ethnic populations, which can largely be explained by racial differences in body weight [11]. Other ethnic factors may also potentially play a role in PK variability of monoclonal antibody therapies, including differences in lymph flow rate, volume and drainage; endocytosis; polymorphisms of the $\mathrm{Fc} \gamma$ receptor gene and neonatal Fc receptor (FcRn) gene, and FcRn-mediated recycling/clearance of immunoglobulin $G$ ( $\operatorname{IgG})$; target levels and target-mediated drug disposition/clearance; and immunogenicity [11]. However, data on these factors are sparse. To assess whether Chinese ethnicity has the potential to influence the PK of belimumab, the current study was undertaken to evaluate the PK, PD, and safety of a single dose of belimumab $10 \mathrm{mg} / \mathrm{kg}$ IV in Chinese patients with SLE.

\section{METHODS}

\section{Study Design}

A phase 1, open-label, single-dose study (GSK study 200909; ClinicalTrials.gov NCT02880852) of IV administration of belimumab in Chinese patients with SLE was conducted between 23 January 2017 and 8 September 2017 at the Huashan Hospital Affiliated to Fudan University, Shanghai, and The First Affiliated Hospital of Suzhou University, Suzhou, China.
The primary objective was to investigate the PK of IV administration of belimumab in Chinese patients with SLE. Secondary objectives evaluated safety, tolerability, and PD. Patients were screened within 30 days prior to dosing and received a single dose of belimumab $10 \mathrm{mg} / \mathrm{kg}$ IV in the study center on day 0. Lyophilized belimumab was reconstituted with sterile water for injection and infused over $1 \mathrm{~h}$. Patients remained in the study center for up to $24 \mathrm{~h}$ post-dose and attended subsequent outpatient visits on days $1,7,14,21,28,42,56$, and 84.

Medications initiated prior to screening could be continued during the study and adjusted as clinically required following receipt of belimumab, except for those listed in Table S1 in the electronic supplementary material. Antihistamine and acetaminophen could be administered prophylactically in patients with a history of allergies or urticaria. All medications taken 60 days before screening and during the study were recorded.

All procedures performed in studies involving human participants were in accordance with the ethical standards of the institutional and/or national research committee and with the 1964 Helsinki declaration and its later amendments or comparable ethical standards. The study was approved by the following institutional review boards: Ethics Committee, Fudan University Huashan Hospital: Approval letter reference-(2016) Clinical Trial Review No. 198 and Ethics Committee, The First Affiliated Hospital of Soochow University: Approval letter reference-(2016) EC review approval No. 009. Informed consent was obtained from all individual participants included in the study.

\section{Study Population}

Eligible patients were male or female, at least 18 years of age at screening, with a clinical diagnosis of SLE according to the American College of Rheumatology classification criteria, and either not taking medication for SLE or receiving a stable SLE treatment regimen for at least 2 months prior to belimumab dosing. Patients were also required to have a confirmed positive 
test for antinuclear antibodies and/or anti-double-stranded deoxyribonucleic acid serum antibodies, and to have a QT duration of less than $450 \mathrm{~ms}$ when corrected for heart rate by Bazett's formula $(\mathrm{QTcB})$ or Fridericia's formula (QTcF) (less than $480 \mathrm{~ms}$ in patients with bundle branch block).

Key exclusion criteria included severe lupus kidney disease (proteinuria greater than $6 \mathrm{~g} / 24 \mathrm{~h}$ ) within 6 months of screening, active central nervous system lupus requiring medical intervention within 6 months of screening, a history of or a positive test at screening for human immunodeficiency virus and/or hepatitis B or C infection, receipt of any B cell targeted therapy or a history of major organ or hematopoietic stem cell/marrow transplant.

\section{Endpoints}

The primary endpoint comprised the following PK parameters: peak serum concentration $\left(C_{\max }\right)$, area under the concentration-time curve (AUC) from time zero (pre-dose) to last time of quantifiable concentration within a patient $\left(\mathrm{AUC}_{0-t}\right)$, AUC from time zero extrapolated to infinity $\left(\mathrm{AUC}_{0-\infty}\right)$, terminal $t_{1 / 2}$, terminal phase rate constant $(\lambda z), \mathrm{CL}$, and $V_{\mathrm{z}}$.

Secondary endpoints for PD parameter estimation were total serum IgG, IgM, and IgA, and various $\mathrm{B}$ cell subsets $\left(\mathrm{CD} 20^{+}, \mathrm{CD} 20^{+} / 27^{+}\right.$memory, $\mathrm{CD} 20^{+} / 27^{-}$naïve, $\mathrm{CD} 20^{+} / 69^{+}$activated, $\mathrm{CD} 20^{+} /$ $138^{+}$plasmacytoid, CD $19^{+} / 27^{\mathrm{BRIGHT}} / 38^{\mathrm{BRIGHT}}$ SLE subset, and $\mathrm{CD}^{-} \mathrm{O}^{-} / 138^{+}$plasma cells), and safety and tolerability [adverse events (AEs), vital signs, 12-lead electrocardiogram, and clinical laboratory safety tests].

\section{Sample Collection and Analysis}

Blood samples for PK analyses were collected into 4-mL dry tubes pre-dose, and at $5 \mathrm{~min}, 1 \mathrm{~h}$, and $24 \mathrm{~h}$ post-dose (the end of the infusion was designated time 0 ), and on days $1,7,14,21,28$, 42,56 , and 84 post-dose. Belimumab serum concentrations were measured in a central laboratory (Covance China) using a validated analytical method based on an electrochemiluminescence immunoassay (MULTI-ARRAY BAFF kit; Meso Scale Discovery, Rockville, USA) as per the manufacturer's instructions, with lower and upper limits of quantification of $100 \mathrm{ng} / \mathrm{mL}$ and $12,800 \mathrm{ng} / \mathrm{mL}$, respectively. For the analysis to be acceptable, no more than one-third of the quality control results were to deviate from the nominal concentration by more than $20 \%$, and at least $50 \%$ of the results from each quality control concentration should be within $20 \%$ of nominal.

PK parameters were derived using non-compartmental analysis and WinNonlin software (version 6.3), based on actual sampling times. $\mathrm{AUC}_{0-t}$ was calculated by a combination of linear and logarithmic trapezoidal methods, used for all incremental trapezoids arising from increasing and decreasing concentrations, respectively. $\mathrm{AUC}_{0-\infty}$ was calculated as $\mathrm{AUC}_{0-t}+C_{t} / \lambda z$, where $C_{t}$ is the last quantifiable concentration; $t_{1 / 2}$ was calculated as $\log _{\mathrm{e}}^{2} / \lambda z ; \lambda z$ was estimated by linear regression of logarithmically transformed concentration versus time data, with only those data points (minimum of three) describing the terminal log-linear decline used in the regression. $\mathrm{CL}$ was calculated as dose/AUC $\mathrm{O}_{-\infty}$, and $V_{\mathrm{z}}$ calculated as $\mathrm{CL} / \lambda z$.

Blood samples for PD analyses were collected pre-dose, and on days 14, 28, 42, 56, and 84 after belimumab dosing and sent to a central laboratory for analysis of total serum IgG, IgM, IgA, and B cell subsets.

AEs were coded using the Medical Dictionary for Regulatory Activities and summarized by System Organ Classes and Preferred Term.

\section{Statistical Analysis}

No formal sample size calculations were undertaken; however, it was planned to enroll 20 patients to obtain data from at least 12 evaluable patients. $\mathrm{PK}, \mathrm{PD}$, and safety endpoints were evaluated using descriptive statistics based on logtransformed data. The between-patient coefficient of variation for PK parameters was approximated using SQRT $(\exp [\mathrm{SD}]-1) \times 100$, where SD is the standard deviation of the log-transformed data. 


\section{RESULTS}

\section{Study Population}

Of the 25 patients screened, 20 were enrolled, received a single dose of belimumab $10 \mathrm{mg} / \mathrm{kg}$ $\mathrm{IV}$, and subsequently completed the study. All patients were included in the PK, PD, and safety analyses. A summary of patient demographics and baseline characteristics is provided in Table S2 in the electronic supplementary material. Briefly, all patients were of East Asian heritage and the majority were female $(n=16$, $80 \%)$, with mean (standard deviation) age of 37.4 (14.1) years and body mass index of 23.1 (3.1) $\mathrm{kg} / \mathrm{m}^{2}$. All patients were positive for antinuclear antibodies, and 14 (70\%) were positive for anti-double-stranded deoxyribonucleic acid antibodies. All patients received at least one concomitant medication during the study, most commonly corticosteroids $(n=18,90 \%)$ and hydroxychloroquine $(n=15,75 \%)$.

\section{Pharmacokinetic Analyses}

Serum concentrations of belimumab peaked at the end of the infusion and declined steadily thereafter, with quantifiable serum concentrations detectable from 5 min up to day 84 postdose (last measurement) (Fig. 1). PK parameters following single IV administration of belimumab are summarized in Table 1.

\section{Pharmacodynamic and Biomarker Analyses}

Slight reductions from baseline in serum levels of IgA, IgG, and IgM were observed up to a maximum of $11.76 \%, 5.42 \%$, and $14.47 \%$, respectively. These values were within the normal range and were not considered clinically significant or drug-related.

The median (range) percentage change from baseline in B cell subsets (absolute counts) by planned time relative to dosing are shown in Fig. 2. The median reduction from baseline for $\mathrm{CD} 19^{+}$B Cells was $-9.41 \%$ on day 28 and $-36.63 \%$ on day 84 , and for CD20 ${ }^{+}$B Cells it was $-10.21 \%$ and $-35.25 \%$ on days 28 and 84 , respectively. The median reduction from baseline on day 14 for naïve $\mathrm{CD} 20^{+} / \mathrm{CD} 27^{-}$B Cells was $-7.72 \%$, which decreased further over time to $-51.94 \%$ on day 84 . Among activated $\mathrm{CD}^{2} 0^{+} / \mathrm{CD} 69^{+} \mathrm{B}$ cells, the median reduction from baseline on day 14 was $-61.10 \%$, and this reduction was sustained through to day 84 ($80.90 \%)$. There was an apparent expansion of $\mathrm{CD} 20^{+} / \mathrm{CD} 27^{+}$memory B cells, with the maximum median increase from baseline observed on day 14 (121.97\%); the corresponding increase on day 84 was $53.78 \%$. The median reductions from baseline for plasmacytoid $\mathrm{CD}^{2} 0^{+} / \mathrm{CD} 138^{+}$and plasma $\mathrm{CD} 20^{-} / \mathrm{CD} 138^{+}$ $B$ cell subsets were $-39.07 \%$ and $-23.20 \%$, respectively, on day 14 , decreasing further over time to $-71.97 \%$ and $-63.66 \%$, respectively, on day 84 . For the $\mathrm{CD} 19^{+} / \mathrm{CD} 27^{+\mathrm{BRIGHT}} /$ CD38+BRIGHT SLE B cell subset, the median reduction from baseline was - $22.55 \%$ on day 14 and $-17.14 \%$ on day 84 .

\section{Safety}

A single dose of belimumab $10 \mathrm{mg} / \mathrm{kg}$ IV was well tolerated among patients with SLE in this study (Table 2). In total, 12 patients (60\%) reported 20 AEs, the majority of which were mild in intensity. Three AEs reported in two patients were moderate in intensity, and no severe AEs were observed. The most frequently reported AEs were upper respiratory tract infection ( $n=5$ patients, 25\%) and hyperglycemia ( $n=2$ patients, $10 \%$ ), with all other AEs reported by a single patient each. Four patients had AEs that were considered by the investigator as possibly drug-related, upper respiratory tract infection in four patients (three mild cases, one moderate case) and mild pharyngitis in one patient, all of which had resolved by the end of the study. A serious $\mathrm{AE}$ (SAE) of moderate upper respiratory tract infection was reported in one patient, who also had an SAE of neutropenia (not considered drug-related). No other SAEs or deaths were reported.

\section{DISCUSSION}

This study was undertaken to investigate the PK and PD properties of belimumab $10 \mathrm{mg} / \mathrm{kg}$ IV in 

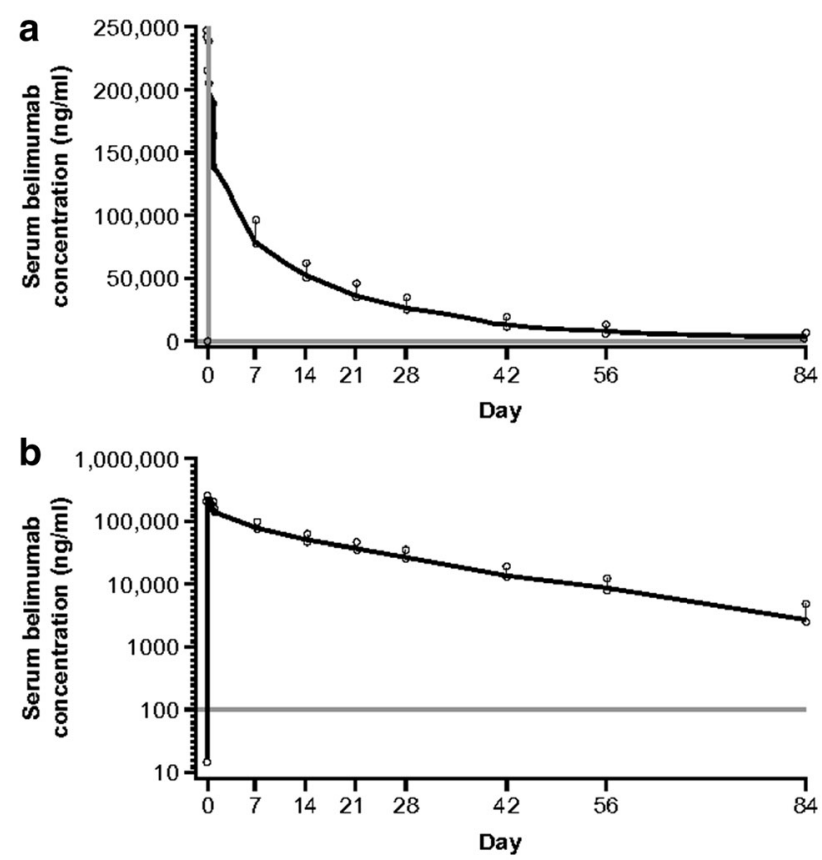

Fig. 1 Mean (SD) serum concentration-time profile of belimumab after a single IV dose $(10 \mathrm{mg} / \mathrm{kg})$ in Chinese patients with SLE $(N=20)$, depicted on a linear and $\mathbf{b}$

semilogarithmic scales. IV intravenous, SD standard deviation, SLE systemic lupus erythematosus

Table 1 Pharmacokinetic parameters of a single dose of belimumab $10 \mathrm{mg} / \mathrm{kg} \mathrm{IV}$ in Chinese patients with SLE $\left(N=20^{\mathrm{a}}\right)$

\begin{tabular}{lll}
\hline Pharmacokinetic parameter & Geometric mean $\mathbf{9 5 \%} \mathbf{C I})$ & Between-patient coefficient of variation \\
\hline$C_{\text {max }}, \mu \mathrm{g} / \mathrm{mL}$ & $221(208,235)$ & 12.8 \\
$\mathrm{AUC}_{0-t}$, day $\cdot \mu \mathrm{g} / \mathrm{mL}$ & $2395(2151,2667)$ & 23.3 \\
$\mathrm{AUC}_{0-\infty}$, day $\cdot \mu \mathrm{g} / \mathrm{mL}$ & $2462(2197,2759)$ & 24.7 \\
$t_{1 / 2}$, days & $14.6(12.5,17.2)$ & 35.4 \\
$\lambda z$, per day & $0.0474(0.0403,0.0557)$ & 35.4 \\
$\mathrm{CL}, \mathrm{mL} /$ day $/ \mathrm{kg}$ & $4.06(3.62,4.55)$ & 24.7 \\
$V_{z}, \mathrm{~mL} / \mathrm{kg}$ & $85.7(77.5,94.8)$ & 21.7 \\
\hline
\end{tabular}

$\lambda z$ terminal rate constant, $A U C_{0-\infty}$ area under the concentration-time curve from time zero (pre-dose) extrapolated to infinity, $A U C_{0-t}$ area under the concentration-time curve from time zero (pre-dose) to last time of quantifiable concentration within a patient, $C I$ confidence interval, $C L$ systemic clearance, $C_{\max }$ maximum plasma concentration, $I V$ intravenous, $S L E$ systemic lupus erythematosus, $t_{1 / 2}$ terminal phase half-life, $V_{z}$ volume of distribution

${ }^{a}$ A pre-dose serum sample from one patient showed a quantifiable concentration of belimumab, possibly attributable to interference during the assay or ex vivo contamination of the sample; therefore, this individual sample was not included in the pharmacokinetic analysis

Chinese patients with SLE, as well as the safety and tolerability of a single dose. After a single $10 \mathrm{mg} / \mathrm{kg}$ IV dose of belimumab, geometric mean $t_{1 / 2}, \mathrm{CL}, V_{\mathrm{z}}, C_{\text {max }}$, and $\mathrm{AUC}_{0-t}$ were 14.6 days, $4.06 \mathrm{~mL} / \mathrm{day} / \mathrm{kg}, 85.7 \mathrm{~mL} / \mathrm{kg}, 221 \mu \mathrm{g} /$ $\mathrm{mL}$, and 2395 day $\mu \mathrm{g} / \mathrm{mL}$, respectively. These 


\section{a}
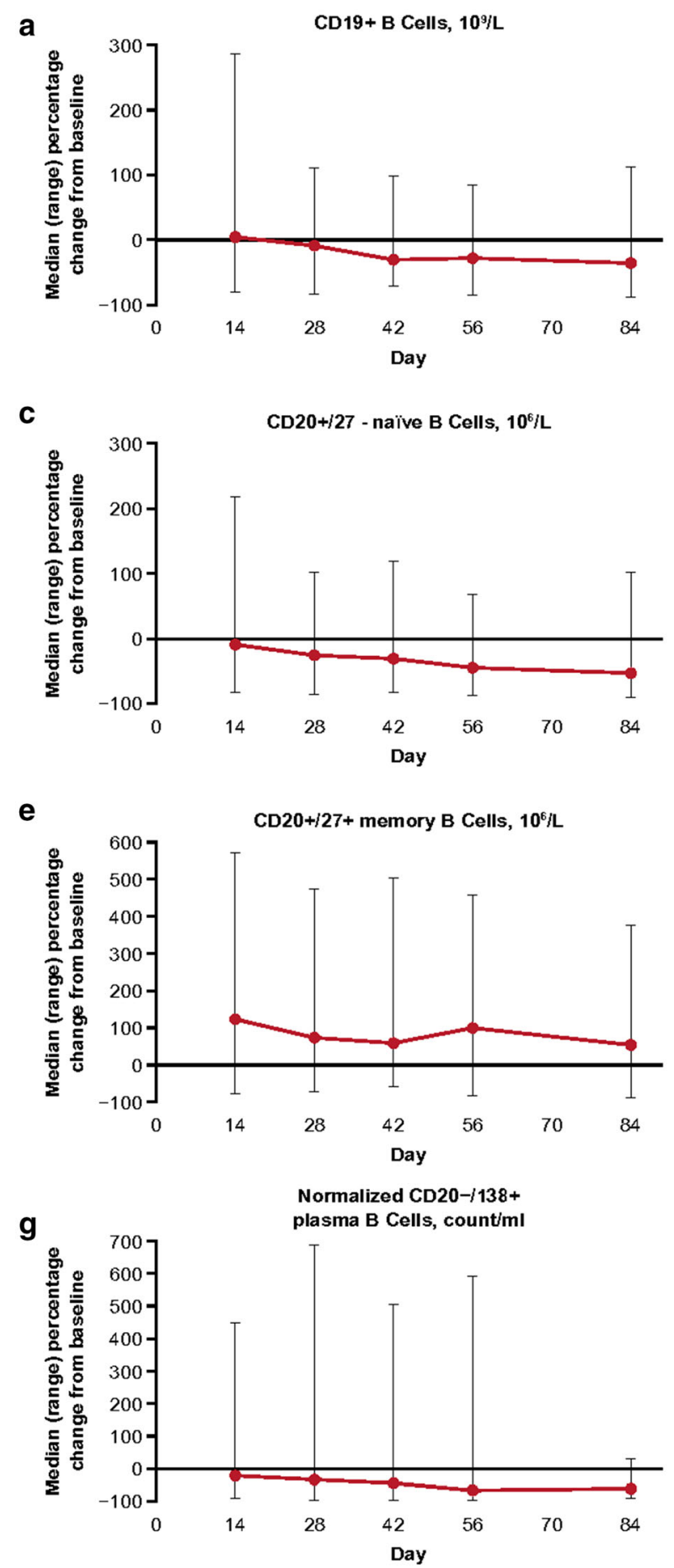
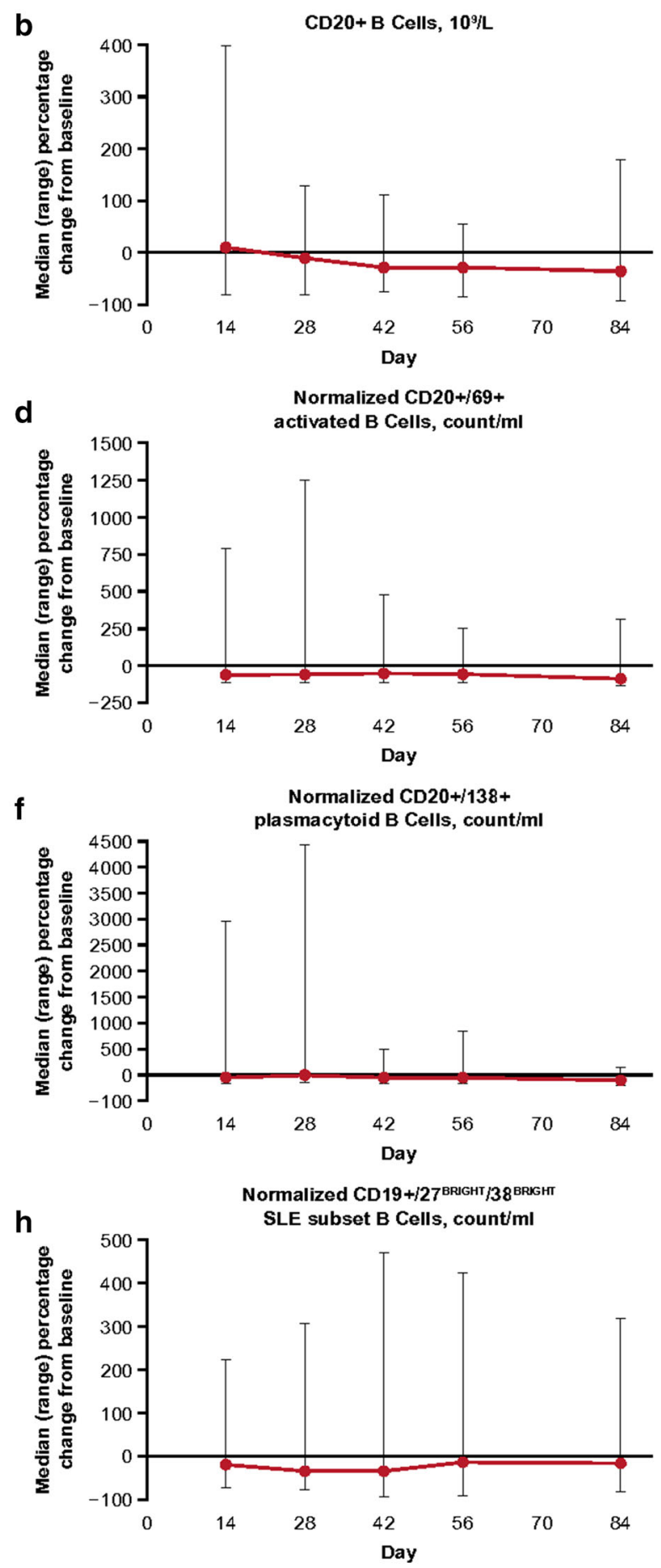

Fig. 2 Percentage change from baseline in B cell subsets in Chinese patients with SLE who received a single dose of belimumab $10 \mathrm{mg} / \mathrm{kg}$ IV $(N=20)$. IV intravenous, SLE systemic lupus erythematosus, CD, cluster of differentiation 
Table 2 Summary of AEs reported in Chinese patients with SLE who received a single dose of belimumab $10 \mathrm{mg} /$ $\operatorname{kg}$ IV $(N=20)$

\begin{tabular}{lc}
\hline AE, $\boldsymbol{n}(\mathbf{\%})$ & $(\boldsymbol{N}=\mathbf{2 0})$ \\
\hline Any AE & $12(60)$ \\
Upper respiratory tract infection & $5(25)$ \\
Hyperglycemia & $2(10)$ \\
Anemia & $1(5)$ \\
Balanoposthitis & $1(5)$ \\
Bone infarction & $1(5)$ \\
Ecchymosis & $1(5)$ \\
Gingivitis & $1(5)$ \\
Hypertension & $1(5)$ \\
Hypokalemia & $1(5)$ \\
Neutropenia & $1(5)$ \\
Osteoarthritis & $1(5)$ \\
Oropharyngeal pain & $1(5)$ \\
Pharyngitis & $1(5)$ \\
Productive cough & $1(5)$ \\
\hline
\end{tabular}

$A E$ adverse event, $I V$ intravenous, $S L E$ systemic lupus erythematosus

results are consistent with $\mathrm{PK}$ values for Japanese patients in a previous study [9], who demonstrated geometric mean $t_{1 / 2}, \mathrm{CL}$, $V_{\mathrm{z}}, C_{\text {max }}$, and $\mathrm{AUC}_{0-t}$ values of 15.7 days, $3.6 \mathrm{~mL} /$ day $/ \mathrm{kg}, \quad 76.2 \mathrm{~mL} / \mathrm{kg}, \quad 223 \mu \mathrm{g} / \mathrm{mL}$, and 2814 day. $\mu \mathrm{g} / \mathrm{mL}$, respectively [9]. The values from Chinese and Japanese patients differ slightly from those of patients in a US study, who showed a slightly higher mean CL $(6.9 \mathrm{~mL} /$ day $/ \mathrm{kg})$, a lower $t_{1 / 2}\left(10.6\right.$ days), $C_{\max }$ $(192 \mu \mathrm{g} / \mathrm{mL})$, and $\mathrm{AUC}_{0-t}(1510 \mathrm{day} \cdot \mu \mathrm{g} / \mathrm{mL})$, and a similar $V_{\mathrm{z}}(86.3 \mathrm{~mL} / \mathrm{kg})$ [10]. The American patients were predominantly of non-Asian ethnicity $(N=14$, of whom 8 were white, 5 were African-American, and 1 was Asian) [10], so these differences may partially reflect a known effect of body size on certain PK parameters (e.g., increased CL with larger body size) [12]; the clinical relevance of these differences is typically low [13]. PK parameters in the present study were also consistent with those of a pooled analysis of the two pivotal phase 3 trials of IV administration of belimumab in patients with SLE: geometric means of $t_{1 / 2}$ of 17.2 18.7 days, CL of $3.24-3.96 \mathrm{~mL} / \mathrm{day} / \mathrm{kg}, V_{\text {ss }}$ of ca. $71-98 \mathrm{~mL} / \mathrm{kg}, \quad C_{\max }$ of $274-360 \mu \mathrm{g} / \mathrm{mL}$, and $\mathrm{AUC}_{0-t}$ of $2357-3419$ day. $\mu \mathrm{g} / \mathrm{mL}$ (ranges based on body weight stratification from less than $54 \mathrm{~kg}$ to $75 \mathrm{~kg}$ or more) [12].

The PK profile observed in the present study is consistent with findings with other IgG1 monoclonal antibodies, which do not experience clinically significant target-mediated disposition [12]. In the present study, the reduction in $\mathrm{CD} 20^{+}, \mathrm{CD} 20^{+} / \mathrm{CD} 27^{-}$naïve, $\mathrm{CD} 20^{+} / \mathrm{CD} 69^{+}$active, $\mathrm{CD} 20^{+} / \mathrm{CD} 138^{+}$plasmacytoid, $\mathrm{CD} 19^{+} / \mathrm{CD} 27^{\mathrm{BRIGHT}} / \mathrm{CD} 38^{\mathrm{BRIGHT}}$ SLE subset, and $\mathrm{CD}_{20}^{-} / \mathrm{CD} 138^{+}$plasma B cells, along with the apparent expansion of $\mathrm{CD}^{+} \mathrm{O}^{+} /$ $\mathrm{CD} 27^{+}$memory B cell concentrations, is consistent with the known ability of belimumab to bind free BLyS and inhibit its function, providing evidence of biological activity [6].

PK samples were collected from patients for 84 days after dosing (i.e., more than 5 half-lives of belimumab) to enable a comprehensive PK profile to be determined following a single IV dose. Although the PK of belimumab $10 \mathrm{mg} / \mathrm{kg}$ IV has not been assessed in healthy volunteers, it has been assessed in patients with mild-tomoderate SLE from Japan [9] and the USA [10]. Therefore, Chinese patients with SLE, rather than healthy Chinese volunteers, were recruited into this study to allow unbiased comparison between Chinese and non-Chinese patients.

The single dose of belimumab $10 \mathrm{mg} / \mathrm{kg}$ IV was well tolerated in Chinese patients with SLE, with no new safety signals detected. Upper respiratory tract infection and pharyngitis were the only AEs considered drug-related, and these had resolved before the end of the study. The safety profile of belimumab in this study was consistent with that in the phase 3 study conducted in Northeast Asia in which patients received belimumab for 48 weeks [8], with upper respiratory tract infection and nasopharyngitis being the most commonly reported AEs. These findings are in keeping with those of other clinical studies of belimumab in patients with SLE, in 
which it was well tolerated when one or two doses of up to $20 \mathrm{mg} / \mathrm{kg}$, or multiple doses of 1 or $10 \mathrm{mg} / \mathrm{kg}$, were administered for up to 76 weeks $[9,10,14]$.

As per the similar PK study of a single belimumab $10 \mathrm{mg} / \mathrm{kg}$ IV dose in Japanese patients with SLE [9], the small sample size and single-dose design preclude a full assessment of the PK, PD, and long-term safety of belimumab in Chinese patients with SLE. A further limitation is that the PD effect after a single dose of belimumab may not be predictive of its PD effects after chronic dosing.

\section{CONCLUSIONS}

In this study undertaken to assess the PK, PD, and safety of a single belimumab $10 \mathrm{mg} / \mathrm{kg}$ IV dose in Chinese patients with SLE, belimumab PK were similar to those observed in other patients with SLE in Japan and the USA (white or African-American). A biological effect was evident, with reductions noted in all tested B cell subsets, except for memory B cells, which expanded. Belimumab was also well tolerated. Taken together with the findings of the phase 3, Northeast Asian study [8], these data support the use of belimumab for the treatment of SLE in Chinese patients.

\section{ACKNOWLEDGEMENTS}

We would like to acknowledge the contribution of Tsung-I Lee and Yucheng Sheng.

Funding. This study (GSK study 200909; NCT02880852) and the Rapid Service Fee were funded by GlaxoSmithKline. All authors had full access to all of the data in this study and take complete responsibility for the integrity of the data and accuracy of the data analysis.

Medical Writing Assistance. Medical writing support was provided by Jennie McLean, PhD, of Fishawack Indicia Ltd, UK, and was funded by GSK.
Authorship. All named authors meet the International Committee of Medical Journal Editors (ICMJE) criteria for authorship for this article, take responsibility for the integrity of the work as a whole, and have given their approval for this version to be published.

Authorship Contributions. Chao Li, Cui Xiong, Jing Zhang, Jian $\mathrm{Wu}$, Liyan Miao, Yinghua Shen, Peiming Ma, Weiguo Wan and $\mathrm{Yu}$ Xue contributed to the study design; Guoying Cao, Jian $\mathrm{Wu}$, Jing Zhang, Liyan Miao, Weiguo Wan and Yu Xue assisted with data acquisition; and Chao Li, Cui Xiong, Jun Dong, Jing Zhang, Yinghua Shen, and Peiming Ma were involved in data analysis or interpretation.

Disclosures. Peiming Ma, Jun Dong, Yinghua Shen and Cui Xiong are employees of GlaxoSmithKline (GSK) and hold stocks and shares in the company. Chao $\mathrm{Li}$ was an employee of GSK at the time of the study and is now an employee of Novartis Pharma, China. Jing Zhang, Weiguo Wan, Liyan Miao, Jian Wu, $\mathrm{Yu}$ Xue and Guoying Cao have nothing to disclose.

Compliance with Ethics Guidelines. All procedures performed in studies involving human participants were in accordance with the ethical standards of the institutional and/or national research committee and with the 1964 Helsinki declaration and its later amendments or comparable ethical standards. The study was approved by the following institutional review boards: Ethics Committee, Fudan University Huashan Hospital: Approval letter reference(2016) Clinical Trial Review No. 198 and Ethics Committee, The First Affiliated Hospital of Soochow University: Approval letter reference(2016) EC review approval No. 009. Informed consent was obtained from all individual participants included in the study.

Data Availability. Within 6 months of this publication, anonymized individual participant data, the annotated case report form, protocol, reporting and analysis plan, data set specifications, raw dataset, analysis-ready dataset and clinical study report will be available for 
research proposals approved by an independent review committee. Proposals should be submitted to www.clinicalstudydatarequest.com. A data access agreement will be required.

Open Access. This article is distributed under the terms of the Creative Commons AttributionNonCommercial 4.0 International License (http://creativecommons.org/licenses/by-nc/4.0/), which permits any noncommercial use, distribution, and reproduction in any medium, provided you give appropriate credit to the original author(s) and the source, provide a link to the Creative Commons license, and indicate if changes were made.

\section{REFERENCES}

1. Di Battista M, Marcucci E, Elefante E, et al. One year in review 2018: systemic lupus erythematosus. Clin Exp Rheumatol. 2018;36:763-77.

2. Mok CC. Epidemiology and survival of systemic lupus erythematosus in Hong Kong Chinese. Lupus. 2011;20:767-71.

3. Mok CC, To $\mathrm{CH}, \mathrm{Ho} \mathrm{LY}, \mathrm{Yu} \mathrm{KL}$. Incidence and mortality of systemic lupus erythematosus in a southern Chinese population, 2000-2006. J Rheumatol. 2008;35:1978-82.

4. Rees F, Doherty M, Grainge MJ, Lanyon P, Zhang $\mathrm{W}$. The worldwide incidence and prevalence of systemic lupus erythematosus: a systematic review of epidemiological studies. Rheumatology (Oxford). 2017;56:1945-61.

5. Wang Z, Wang Y, Zhu R, et al. Long-term survival and death causes of systemic lupus erythematosus in China: a systemic review of observational studies. Medicine (Baltimore). 2015;94:e794.

6. GlaxoSmithKline. Belimumab US prescribing information. https://www.accessdata.fda.gov/drugsatfda_ docs/label/2018/125370s062,761043s002lbl.pdf. Accessed 1 Jul 2019.

7. FDA. News release. FDA approves first treatment for paediatric patients with lupus. 2019. https://www. fda.gov/news-events/press-announcements/fda-app roves-first-treatment-pediatric-patients-lupus. Accessed 1 Jul 2019.

8. Zhang F, Bae SC, Bass D, et al. A pivotal phase III, randomised, placebo-controlled study of belimumab in patients with systemic lupus erythematosus located in China, Japan and South Korea. Ann Rheum Dis. 2018;77:355-63.

9. Yamada M, Akita M, Nakagawa T, Takahashi N, Endo A, Yoshida P. Safety, tolerability, pharmacokinetics and pharmacodynamics of belimumab in Japanese patients with mild-to-moderate systemic lupus erythematosus. J Drug Assess. 2013;2: $40-8$.

10. Furie R, Stohl W, Ginzler EM, et al. Biologic activity and safety of belimumab, a neutralizing anti-Blymphocyte stimulator (BLyS) monoclonal antibody: a phase I trial in patients with systemic lupus erythematosus. Arthritis Res Ther. 2008;10:R109.

11. Gill KL, Machavaram KK, Rose RH, Chetty M. Potential sources of inter-subject variability in monoclonal antibody pharmacokinetics. Clin Pharmacokinet. 2016;55:789-805.

12. Struemper H, Chen C, Cai W. Population pharmacokinetics of belimumab following intravenous administration in patients with systemic lupus erythematosus. J Clin Pharmacol. 2013;53:711-20.

13. Keizer RJ, Huitema AD, Schellens JH, Beijnen JH. Clinical pharmacokinetics of therapeutic monoclonal antibodies. Clin Pharmacokinet. 2010;49: 493-507.

14. Navarra SV, Guzman RM, Gallacher AE, et al. Efficacy and safety of belimumab in patients with active systemic lupus erythematosus: a randomised, placebo-controlled, phase 3 trial. Lancet. 2011;377: 721-31. 